Revista Brasil. Bot., V.34, n.3, p.431-446, jul.-set. 2011

\title{
An overview of invasive plants in Brazil
}

\author{
RAFAEL DUDEQUE ZENNI ${ }^{1,2}$ and SÍLVIA RENATE ZILLER ${ }^{1}$
}

(received: June 22, 2010; accepted: August 17, 2011)

\begin{abstract}
An overview of invasive plants in Brazil). Alien plants are known to occur in Brazil since the $18^{\text {th }}$ century when African grasses started to be recorded in pastures near Rio de Janeiro. In the beginning of the $19^{\text {th }}$ century two royal decrees (July, 1809 and July, 1810) offered grants and tax exemption to everyone who would introduce plants of economic value. Nowadays, there are 117 plant species recognized as invasive or established and with invasive potential in Brazil and an unknown number of introduced plant species. Some of the most pervasive invasive species are Artocarpus heterophyllus Lam. and Hedychium coronarium König in tropical ombrophilous forest, Hovenia dulcis Thunb. in subtropical ombrophilous forest and subtropical semi-deciduous forest, Pinus taeda L. and Pinus elliottii Engelm. in subtropical ombrophilous forest and steppe, Prosopis juliflora (Sw.) DC. in stepic-savanna, Tecoma stans (L.) Juss. ex Kunth in tropical and subtropical semi-deciduous forest, Melinis minutiflora P. Beauv. in the Brazilian savannas, and Eragrostis plana Nees in the steppe. The purpose of this article is to fill a knowledge gap on alien species that are invasive in Brazil and where they are invading by summarizing data obtained by joint efforts of the Hórus Institute for Environmental Conservation and Development, The Nature Conservancy (TNC), the Inter-American Biodiversity Information Network (IABIN) invasive species thematic network (I3N), and the Brazilian Ministry of Environment (MMA) in the last six years.
\end{abstract}

Key words - biological invasions, database, invasive alien plants

RESUMO - (Visão geral das plantas exóticas invasoras no Brasil). Alertas sobre espécies exóticas existem no Brasil desde o século XVIII, quando gramíneas africanas começaram a ser registradas em pastagens próximas ao Rio de Janeiro. No início do século XIX dois decretos reais (em julho de 1809 e julho de 1810) ofereciam bônus e isenção de impostos para todos que introduzissem plantas de valor econômico. Atualmente, há 117 espécies de plantas exóticas reconhecidas como estabelecidas com potencial invasor ou invasoras no Brasil, e um número desconhecido de plantas introduzidas. As plantas exóticas invasoras mais relevantes são Artocarpus heterophyllus Lam. e Hedychium coronarium König na floresta ombrófila densa, Hovenia dulcis Thunb. na floresta ombrófila mista e floresta estacional semidecidual do rio Paraná, Pinus taeda L. e Pinus elliottii Engelm. na floresta ombrófila mista e na estepe, Prosopis juliflora (Sw.) DC. na savana-estépica, Tecoma stans (L.) Juss. ex Kunth nas florestas estacionais semideciduais e deciduais, Melinis minutiflora P. Beauv. na savana e Eragrostis plana Nees na estepe. Este artigo tem como objetivo ajudar a preencher uma lacuna de informação sobre espécies exóticas invasoras no Brasil e os locais onde invadem, apresentando um resumo dos dados obtidos no banco de dados de espécies exóticas invasoras no Brasil criado e mantido nos últimos seis anos por um esforço conjunto do Instituto Hórus de Desenvolvimento e Conservação Ambiental, da The Nature Conservancy (TNC), da Rede Interamericana de Informações sobre a Biodiversidade (IABIN) por meio da rede temática de Espécies Invasoras (I3N) e do Ministério de Meio Ambiente do Brasil (MMA).

Palavras-chave - banco de dados, invasões biológicas, plantas exóticas invasoras

\section{Introduction}

Invasive alien species are one of the most severe threats to the conservation of native species, communities and ecosystems (Vitousek et al. 1996, Wilcove et al. 1998, Traveset \& Richardson 2006) and require urgent attention in many parts of the world (Mack et al. 2000). However, when data is lacking on which species are invading and where they are invading conservation planning strategies and public awareness are limited and ineffective. A critical first step to raise awareness and increase conservation efficiency is an invasive species

\footnotetext{
1. Instituto Hórus de Desenvolvimento e Conservação Ambiental, Servidão Cobra Coral, 111, 88063-513 Florianópolis, SC, Brazil.

2._Corresponding author: rafaeldz@gmail.com
}

assessment based on existing information (Wittenberg \& Cock 2001).

Brazil has the highest plant diversity in the world with two biodiversity hotspots recognized for conservation priorities: Cerrado (Savanna) and Atlantic Forest (Myers et al. 2000). More than half of the country ( $c a .8 .5$ million $\mathrm{km}^{2}$ was originally covered by Amazon Forest, the world's largest tropical forest, while the other half was covered by Atlantic forests, steppes, savannas, wetlands and semi-arid ecosystems (Veloso et al. 1992). However, little is known about the threats posed by invasive alien species to biodiversity and natural resources within these ecosystems.

The earliest records of an invasive alien plant species in Brazil is of African grasses in pastures near Rio de Janeiro (South-Eastern Brazil) from the early $18^{\text {th }}$ 
century. Of these first recorded grasses several species are still invasive today such as Urochloa maxima (Jacq.) RD. Webster, Hyparrhenia rufa (Nees) Stapf, Urochloa mutica (Forssk.) T.Q. Nguyen, and Melinis minutiflora P. Beauv. (Dean 1996). Data on the source of these introductions is often lacking (i.e. deliberate introductions for animal forage or accidental introductions via dry ballast or other vectors), but these species are currently used as forage grasses, with some of them subjected to genetic improvements (see Cook et al. 2005).

Early in the $19^{\text {th }}$ century two royal decrees (July, 1809 and July, 1810) offered grants and tax exemptions to everyone who introduced plants with economic value (Dean 1996). Probably due to incentives like these, the French naturalist Auguste de Saint-Hilaire recorded a great diversity of plants upon his visit to São Paulo in 1818. Most of the species he wrote about were brought from Europe but native to many different parts of the world. Among them were the currently invasive species Sechium edule (Jacq.) Sw., Artocarpus heterophyllus Lam., Elaeis guineensis Jacq., and Ricinus communis L. (Dean 1996). Saint-Hilaire also documented the spread of Melinis minutiflora in the Cerrado (Savanna) in 1824 (Lima 2002).

Currently, despite the existence of extensive number of global and regional databases and information networks on invasive species (Meyerson \& Mooney 2007), information concerning invasive alien species in Brazil is largely unavailable in the scientific literature (Petenon \& Pivello 2008). The purpose of this article is to fill a knowledge gap on species that are invasive in Brazil and where they are invading by summarizing data obtained by joint efforts of the Hórus Institute for Environmental Conservation and Development, The Nature Conservancy (TNC), the Inter-American Biodiversity Information Network (IABIN) invasive species thematic network (I3N), and the Brazilian Ministry of Environment (MMA) in the last six years.

\section{Material and methods}

The current article is an overview assessment of invasive plants in Brazil that attempts to summarize existing information gathered from field observations, interviews and literature reviews in the last six years. The data used for compiling a species list was gathered from the I3N Brazil (IABIN Invasive Species thematic network) database (I3N Brazil 2010). This database contains occurrence records for non-native species which are already invasive or have invasive potential in Brazil, but does not provide a full record of the habitats where the species are found. Invasive species' occurrences are organized by phytophysiognomy using the nomenclature proposed by Veloso et al. (1992), in Portuguese, and by physiognomic-ecological classes following UNESCO (1973), in English. These are the physiognomies where the exotic species are considered invasive in Brazil and, on times, species also native to Brazil are considered invasive in physiognomies where the species are not known to occur historically and naturally. The data are available for public consultation and open to criticism and review. The present overview only mentions invasive species in natural habitats, and does not consider data from urban or suburban areas.

The species in the I3N database are classified as invasive based on their aptitude to establish and spread in a new habitat without direct human assistance after their introduction, while introductions are almost always likely mediated by human assistance, deliberate or accidental. The presence of a species in the database, and therefore in this overview, does not provide relationship to direct impacts or threats. We verified species for their history of invasion elsewhere when they were merely listed as established in Brazil.

Local references are related to municipalities or geographical references and to phytophysiognomy according to the Brazilian Classification System for Vegetation (Veloso et al. 1992). The phytophysiognomies were than matched to the physiognomic-ecological classes (UNESCO 1973) as the former is an adaptation of the later. We used GIS (Geographic Information System) techniques to merge points of occurrence in the database with corresponding ecoregions (sensu Olson et al. 2001). We chose not to directly translate the Brazilian classification system for vegetation to English because the translated names would not fully enclose the concepts behind each class and thus could potentially disconnect the information from its primary source.

We filtered the data to select only invasion and establishment records and to remove occurrences not related to municipalities, as well as records of plants in urban and suburban areas. The status of "established" refers to species which are reproducing locally, and "invasive" refers to species that are reproducing and spreading beyond their points of introduction. Although the presence of species was always confirmed by checking the source (i.e. a specialist, a scientific publication, or a list of plants), we also used field observations, interviews and literature reviews from the last six years to compile a series of informative case studies that collect most of the knowledge available on the impacts of invasive alien plants in Brazil.

\section{Results}

The alien plant species known to invade natural habitats in Brazil are listed in table 1. There were 3,320 database records for "invasion" or "establishment" (figure 1) of 117 different plant species (table 1) in the I3N Brazil database as of December, 2010.

The invasive plants with the greatest number of occurrences recorded are Urochloa decumbens (265 records), Ricinus communis (253 records), Tecoma 
Table 1. Invasive alien plants records for the physiognomic-ecological classes (UNESCO 1973) and ecoregion (Olson et al. 2001) in Brazil. Data is from the I3N Brazil database at www.institutohorus.org.br. We gathered location references in the database from field observations, interviews and literature reviews from 2003 until 2008. Locations are mostly related to municipalities or geographical reference points. Phytophysiognomies with no correspondence in the original physiognomic-ecological classes are marked with *.

\begin{tabular}{ll}
\hline Family/Species & Physiognomic-ecological class \\
\hline $\begin{array}{l}\text { ACANTHACEAE } \\
\text { Thunbergia alata } \text { Bojer ex Sims }\end{array}$ & $\begin{array}{l}\text { Tropical and subtropical semi-deciduous } \\
\text { forest, Tropical ombrophilous forest } \\
\text { Tropical and subtropical semi-deciduous } \\
\text { forest, Tropical ombrophilous forest }\end{array}$ \\
$\begin{array}{l}\text { AGAVACEAE } \\
\text { Agave sisalana } \text { Perrine ex Engelm. }\end{array}$ & $\begin{array}{l}\text { Savanna (or Brazilian savanna), Steppic- } \\
\text { savanna* }\end{array}$ \\
$\begin{array}{l}\text { ANACARDIACEAE } \\
\text { Mangifera indica } \text { L. }\end{array}$ & $\begin{array}{l}\text { Tropical and subtropical deciduous forest, } \\
\text { Tropical and subtropical semi-deciduous } \\
\text { forest, Evergreen broad-leaved woodland, } \\
\text { Tropical ombrophilous forest, Mangrove } \\
\text { forest, Salt meadow, Savanna (or Brazilian } \\
\text { savanna), Steppic-savanna* }\end{array}$ \\
\end{tabular}

APIACEAE

Ammi majus L.

Centella asiatica (L.) Urb.

ARECACEAE

Archontophoenix cunninghamiana

H. Wendl. \& Drude

Elaeis guineensis Jacq.

Livistona chinensis (Jacq.) R. Br. ex Mart.

ASCLEPIADACEAE

Calotropis gigantea (L.) W. T. Aiton

Calotropis procera (Aiton) W. T.

Aiton

Cryptostegia grandiflora $\mathrm{R}$. Br.

ASTERACEAE

Chrysanthemum myconis L.

Cirsium vulgare (Savi) Ten.

Senecio madagascariensis Poir.

BALSAMINACEAE

Impatiens walleriana Hook. f.

\section{Steppe}

Tropical and subtropical semi-deciduous forest, Subtropical ombrophilous forest

Tropical and subtropical semi-deciduous forest, Tropical ombrophilous forest

Evergreen broad-leaved woodland, Tropical ombrophilous forest, Mangrove forest, Salt meadow

Tropical ombrophilous forest

Salt meadow, Steppic-savanna*

Tropical and subtropical deciduous forest, Tropical and subtropical semi-deciduous forest, Salt meadow, Savanna (or Brazilian savanna), Steppic-savanna*

Steppic-savanna*

Steppic-savanna*

Steppe, Tropical ombrophilous forest, Subtropical ombrophilous forest

Steppe

Steppe, Tropical and subtropical semideciduous forest, Tropical ombrophilous forest, Subtropical ombrophilous forest, Savanna (or Brazilian savanna)
Ecoregion

Bahia coastal forests, Alto Paraná Atlantic forests, Serra do Mar coastal forests

Bahia coastal forests, Serra do Mar coastal forests

Cerrado, Caatinga, Pernambuco interior forests

Caatinga, Bahia coastal forests, Alto Paraná Atlantic forests, Pernambuco interior forests, Southern Atlantic mangroves, Serra do Mar coastal forests, Dry Chaco, Cerrado

Uruguayan savanna, Alto Paraná Atlantic forests

Bahia coastal forests, Cerrado

Alto Paraná Atlantic forests, Serra do Mar coastal forests

Southwest Amazon moist forests, PurusMadeira moist forests, Pernambuco interior forests, Bahia coastal forests, Southern Atlantic mangroves, Caatinga

Bahia coastal forests

Caatinga

Caatinga, Bahia coastal forests, Atlantic dry forests, Dry Chaco, Southern Atlantic mangroves

Caatinga

Caatinga

Uruguayan savanna, Alto Paraná Atlantic forests, Araucaria moist forests

Uruguayan savanna

Cerrado, Araucaria moist forests, Bahia interior forests, Bahia coastal forests, Alto Paraná Atlantic forests, Serra do Mar coastal forests 
continuation

\begin{tabular}{ll}
\hline Family/Species & Physiognomic-ecological class \\
\hline BIGNONIACEAE & \\
Spathodea campanulata P. Beauv. & $\begin{array}{l}\text { Ecotone Savanna (or Brazilian savanna) } \\
\text { - Deciduous or semi-deciduous forest, } \\
\text { Tropical and subtropical deciduous forest, } \\
\text { Tropical and subtropical semi-deciduous } \\
\text { forest, Tropical ombrophilous forest }\end{array}$ \\
Ecotone Steppe - Deciduous or semi- \\
deciduous forest, Ecotone Savanna (or \\
Brazilian savanna) - Deciduous or semi- \\
deciduous forest, Steppe, Tropical and \\
subtropical deciduous forest, Tropical \\
and subtropical semi-deciduous forest, \\
Tropical ombrophilous forest, Subtropical \\
ombrophilous forest, Salt meadow, \\
Savanna (or Brazilian savanna)
\end{tabular}

CACTACEAE

Opuntia ficus-indica (L.) Mill.

Tropical and subtropical semi-deciduous forest, Salt meadow, Steppic-savanna*

CAMPANULACEAE

Hippobroma longiflora (L.) G. Don

CAPRIFOLIACEAE

Lonicera japonica Thunb. ex Murray

CASUARINACEAE

Casuarina equisetifolia L.

Tropical ombrophilous forest

Ecoregion

Alto Paraná Atlantic forests, Bahia coastal forests, Serra do Mar coastal forests, Araucaria moist forests

Uruguayan savanna, Alto Paraná Atlantic forests, Cerrado, Araucaria moist forests, Bahia coastal forests, Serra do Mar coastal forests, Atlantic Coast restingas, Bahia interior forests

Caatinga, Bahia coastal forests

Tropical and subtropical semi-deciduous forest, Tropical ombrophilous forest, Subtropical ombrophilous forest, Salt meadow, Savanna (or Brazilian savanna)

Steppe, Tropical and subtropical semideciduous forest, Tropical ombrophilous forest, Salt meadow, Savanna (or Brazilian savanna), Steppic-savanna*

\section{COMBRETACEAE}

Terminalia catappa $\mathrm{L}$.

Tropical and subtropical semi-deciduous forest, Tropical ombrophilous forest, Mangrove forest, Salt meadow

\section{COMMELINACEAE}

Tradescantia zebrina Heynh.

\section{CUCURBITACEAE}

Sechium edule (Jacq.) Sw.

Steppe, Tropical and subtropical semideciduous forest, Tropical ombrophilous forest, Subtropical ombrophilous forest

Tropical ombrophilous forest

\section{CUPRESSACEAE}

Cupressus lusitanica Mill.

CYPERACEAE

Cyperus rotundus $\mathrm{L}$.

Steppe

Tropical and subtropical semi-deciduous forest, Salt meadow, Alpine meadows, Steppic-savanna*

Tropical ombrophilous forest
Araucaria moist forests, Southern Atlantic mangroves, Pernambuco coastal forests, Bahia coastal forests, Serra do Mar coastal forests, Atlantic dry forests, Caatinga, Bahia interior forests

Bahia coastal forests, Bahia interior forests, Serra do Mar coastal forests

Cerrado, Bahia coastal forests, Serra do Mar coastal forests, Araucaria moist forests

Alto Paraná Atlantic forests, Araucaria moist forests, Serra do Mar coastal forests

Cerrado

Bahia interior forests, Bahia coastal forests, Araucaria moist forests, Caatinga, Pernambuco interior forests, Pernambuco coastal forests

Bahia coastal forests 


\begin{tabular}{ll}
\hline Family/Species & Physiognomic-ecological class \\
\hline EUPHORBIACEAE & \\
Aleurites moluccanus (L.) Willd. & Steppic-savanna* \\
Euphorbia tirucalli L. & Tropical ombrophilous forest \\
Hura crepitans L. & Tropical and subtropical semi-deciduous \\
& forest \\
Ricinus communis L. & Ecotone Steppe - Deciduous or semi- \\
& deciduous forest, Ecotone Tropical \\
& ombrophilous forest - Subtropical \\
& ombrophilous forest, Ecotone Savanna (or \\
& Brazilian savanna) - Deciduous or semi- \\
& deciduous forest, Ecotone Savanna (or \\
& Brazilian savanna)-Savanna (or Brazilian \\
& savanna) Estépica, Steppe, Tropical and \\
& subtropical deciduous forest, Tropical \\
& and subtropical semi-deciduous forest, \\
& Tropical ombrophilous forest, Mangrove \\
forest, Salt meadow, Savanna (or Brazilian & savanna), Steppic-savanna*
\end{tabular}

\section{FABACEAE}

Acacia auriculiformis A. Cunn. ex Benth.

Acacia farnesiana (L.) Willd.

Acacia holosericea A. Cunn. ex G. Don

Acacia longifolia (Andrews) Willd.

Acacia mangium Willd.

Acacia mearnsii De Wild.

Acacia podalyriifolia A. Cunn. ex

G. Don

Albizia falcata (L.) Backer ex Merr.
Tropical and subtropical semi-deciduous meadow

Tropical and subtropical deciduous forest

Tropical and subtropical semi-deciduous forest, Mangrove forest

Ecotone Savanna (or Brazilian savanna)

- Deciduous or semi-deciduous forest, Ecotone Savanna (or Brazilian savanna) Savanna (or Brazilian savanna) Estépica, Salt meadow, Steppic-savanna*

Ecotone Savanna (or Brazilian savanna) - Ombrophilous forest, Tropical and subtropical semi-deciduous forest, Tropical ombrophilous forest, Swamp, Salt meadow, Savanna (or Brazilian savanna), Steppic-savanna*

Ecotone Steppe - Deciduous or semideciduous forest, Ecotone Tropical ombrophilous forest - Subtropical ombrophilous forest, Ecotone Savanna (or Brazilian savanna) - Subtropical ombrophilous forest, Steppe, Steppe Parque, Tropical and subtropical deciduous forest, Tropical ombrophilous forest, Subtropical ombrophilous forest, Salt meadow, Savanna (or Brazilian savanna), Steppic-savanna*

Steppe, Salt meadow

Tropical ombrophilous forest, Mangrove forest forest, Tropical ombrophilous forest, Salt

\author{
Ecoregion
}

Caatinga

Bahia coastal forests

Bahia coastal forests

Uruguayan savanna, Araucaria moist forests, Caatinga, Cerrado, Alto Paraná Atlantic forests, Atlantic Coast restingas, Bahia interior forests, Southern Atlantic mangroves, Bahia coastal forests, Serra do Mar coastal forests, Pernambuco interior forests, Atlantic dry forests, Dry Chaco

Bahia coastal forests, Bahia interior forests, Serra do Mar coastal forests

Cerrado

Bahia coastal forests, Bahia interior forests

Caatinga, Serra do Mar coastal forests, Uruguayan savanna, Atlantic Coast restingas

Guianan savanna, Japurá-Solimoes-Negro moist forests, Bahia coastal forests, Bahia interior forests, Guianan moist forests, Amazon-Orinoco-Southern Caribbean mangroves, Serra do Mar coastal forests, Caatinga

Uruguayan savanna, Araucaria moist forests, Cerrado, Alto Paraná Atlantic forests, Serra do Mar coastal forests, Caatinga

Cerrado, Serra do Mar coastal forests

Bahia coastal forests 
continuation

\begin{tabular}{ll}
\hline Family/Species & Physiognomic-ecological class \\
\hline Clitoria fairchildiana R.A. Howard & $\begin{array}{l}\text { Tropical and subtropical semi-deciduous } \\
\text { forest, Tropical ombrophilous forest } \\
\text { Tropical and subtropical semi-deciduous } \\
\text { forest, Evergreen broad-leaved woodland }\end{array}$ \\
Crotalaria spectabilis Roth & Evergreen broad-leaved woodland \\
Leucaena leucocephala (Lam.) de & $\begin{array}{l}\text { Ecotone Savanna (or Brazilian savanna) } \\
\text { Wit }\end{array}$ \\
& $\begin{array}{l}\text { Tropical and subtropical semi-deciduous } \\
\text { forest, Tropical ombrophilous forest, } \\
\text { Mangrove forest, Salt meadow, Savanna } \\
\text { (or Brazilian savanna), Steppic-savanna* }\end{array}$ \\
\end{tabular}

Parkinsonia aculeata $\mathrm{L}$.

Prosopis juliflora (Sw.) DC.

Steppic-savanna*

Tropical and subtropical semi-deciduous forest, Tropical ombrophilous forest, Savanna (or Brazilian savanna), Steppicsavanna*

Pueraria phaseoloides (Roxb.) Benth.

Steppe, Evergreen broad-leaved woodland, Tropical ombrophilous forest

Ulex europaeus L.

Ecotone Ombrophilous forest Deciduous or semi-deciduous forest, Steppe, Subtropical ombrophilous forest, Salt meadow

\section{IRIDACEAE}

Crocosmia $\times$ crocosmiiflora

(Lemoine) N.E. Br.

\section{LAURACEAE}

Persea americana Mill.

\section{LILIACEAE}

Asparagus setaceus (Kunth) Jessop

Curculigo capitulata (Lour.) Kuntze

Dracaena fragrans (L.) Ker Gawl.

Ophiopogon japonicus (L. f.) Ker

Gawl.

Sansevieria trifasciata Prain

LOMARIOPSIDACEAE

Nephrolepis cordifolia (L.) C. Presl

MALVACEAE

Sterculia foetida L.

Thespesia populnea (L.) Sol. ex Corrêa

Tropical and subtropical semi-deciduous forest, Subtropical ombrophilous forest, Savanna (or Brazilian savanna)

Tropical and subtropical semi-deciduous forest, Tropical ombrophilous forest

Steppe, Tropical and subtropical semideciduous forest, Salt meadow

Tropical ombrophilous forest

Tropical and subtropical semi-deciduous forest, Tropical ombrophilous forest, Salt meadow

Steppe, Subtropical ombrophilous forest

Tropical ombrophilous forest, Salt meadow

Steppe

Tropical and subtropical semi-deciduous forest

Mangrove forest
Ecoregion

Bahia coastal forests

Bahia coastal forests, Purus-Madeira moist forests, Southwest Amazon moist forests Purus-Madeira moist forests, Southwest Amazon moist forests

Alto Paraná Atlantic forests, Caatinga, Bahia coastal forests, Pernambuco interior forests, Cerrado, Bahia interior forests, Serra do Mar coastal forests, Amazon-OrinocoSouthern Caribbean mangroves, Southern Atlantic mangroves, Pernambuco coastal forests, Guianan savanna, Atlantic dry forests, Araucaria moist forests, Dry Chaco, Pantanal, Maranhão Babaçu forests Caatinga, Pernambuco interior forests Caatinga, Southern Atlantic mangroves, Cerrado

Uruguayan savanna, Southwest Amazon moist forests, Japurá-Solimoes-Negro moist forests, Uatuma-Trombetas moist forests, Guianan moist forests

Alto Paraná Atlantic forests, Araucaria moist forests, Cerrado, Uruguayan savanna, Atlantic Coast restingas

Bahia interior forests, Bahia coastal forests, Alto Paraná Atlantic forests, Araucaria moist forests

Bahia coastal forests

Uruguayan savanna

Serra do Mar coastal forests

Bahia coastal forests, Serra do Mar coastal forests, Araucaria moist forests

Araucaria moist forests

Bahia coastal forests

Cerrado

Bahia coastal forests, Bahia interior forests Southern Atlantic mangroves

continue 
Family/Species

Urena lobata L.

MELIACEAE

Azadirachta indica A. Juss.

Melia azedarach $\mathrm{L}$.

MORACEAE

Artocarpus heterophyllus Lam.

Morus nigra L.

\section{MUSACEAE}

Musa ornata Roxb.

Musa rosacea Jacq.

MYRTACEAE

Eucalyptus robusta $\mathrm{Sm}$.

Eugenia malaccensis L. ${ }^{1}$

Psidium guajava $\mathrm{L}$.

Syzygium cumini (L.) Skeels
Physiognomic-ecological class

Evergreen broad-leaved woodland, Tropical ombrophilous forest, Savanna (or Brazilian savanna)

Ecotone Savanna (or Brazilian savanna) - Deciduous or semi-deciduous forest, Ecotone Savanna (or Brazilian savanna) - FP de Influência Mar, Tropical and subtropical semi-deciduous forest, Evergreen broad-leaved woodland, Tropical ombrophilous forest, Mangrove forest, Salt meadow, Savanna (or Brazilian savanna), Steppic-savanna*

Ecotone Savanna (or Brazilian savanna) - Ombrophilous forest, Steppe, Tropical and subtropical deciduous forest, Tropical and subtropical semi-deciduous forest, Tropical ombrophilous forest, Subtropical ombrophilous forest, Savanna (or Brazilian savanna)

Tropical and subtropical semi-deciduous forest, Tropical ombrophilous forest, Salt meadow, Savanna (or Brazilian savanna), Steppic-savanna*

Ecotone Tropical ombrophilous forest Subtropical ombrophilous forest, Tropical and subtropical deciduous forest, Tropical and subtropical semi-deciduous forest, Tropical ombrophilous forest, Mangrove forest, Savanna (or Brazilian savanna)

Tropical ombrophilous forest, Steppicsavanna*

Tropical ombrophilous forest

Campinarana*, Savanna (or Brazilian savanna), Steppic-savanna*

Tropical ombrophilous forest

Ecotone Tropical ombrophilous forest - Subtropical ombrophilous forest, Ecotone Savanna (or Brazilian savanna) - Ombrophilous forest, Steppe, Tropical and subtropical semi-deciduous forest, Evergreen broad-leaved woodland, Tropical ombrophilous forest, Salt meadow, Savanna (or Brazilian savanna) Tropical and subtropical semi-deciduous forest, Tropical ombrophilous forest, Salt meadow, Savanna (or Brazilian savanna), Steppic-savanna*
Ecoregion

Madeira-Tapajós moist forests, Serra do Mar coastal forests, Cerrado

Caatinga, Pernambuco interior forests, Pernambuco coastal forests, Bahia coastal forests, Madeira-Tapajós moist forests, Southwest Amazon moist forests, Southern Atlantic mangroves, Maranhão Babaçu forests, Atlantic dry forests, Atlantic Coast restingas

Chiquitano dry forests, Cerrado, Araucaria moist forests, Alto Paraná Atlantic forests, Serra do Mar coastal forests

Bahia coastal forests, Southern Atlantic mangroves, Bahia interior forests, Serra do Mar coastal forests, Araucaria moist forests, Caatinga

Araucaria moist forests, Serra do Mar coastal forests, Alto Paraná Atlantic forests, Bahia coastal forests

Araucaria moist forests, Caatinga

Araucaria moist forests

Cerrado, Atlantic Coast restingas

Bahia coastal forests

Araucaria moist forests, Alto ParanáAtlantic forests, Serra do Mar coastal forests, Cerrado, Bahia coastal forests, Southwest Amazon moist forests, Pernambuco interior forests, Bahia interior forests

Bahia coastal forests, Alto Paraná Atlantic forests, Bahia interior forests, Araucaria moist forests, Cerrado, Atlantic dry forests 
continuation

Family/Species

OLEACEAE

Ligustrum japonicum Thunb.

Ligustrum lucidum W. T. Aiton

Ligustrum vulgare L.

PINACEAE

Pinus caribaea Morelet

Pinus elliottii Engelm.

Pinus oocarpa Schiede ex Schltdl.

Pinus patula Schltdl. \& Cham.

Pinus taeda L.

\section{PITTOSPORACEAE}

Pittosporum undulatum Vent.

\section{POACEAE}

Andropogon gayanus Kunth

Arundo donax L.

Bambusa textilis McClure

Bambusa vulgaris Schrad. ex J. C. Wendl.

Cenchrus ciliaris L.
Physiognomic-ecological class

Ecoregion

Steppe, Subtropical ombrophilous forest Steppe, Tropical and subtropical semi-deciduous forest, Subtropical ombrophilous forest

Subtropical ombrophilous forest

Ecotone Savanna (or Brazilian savanna) - Ombrophilous forest, Tropical and subtropical semi-deciduous forest, Tropical ombrophilous forest, Salt meadow

Ecotone Steppe - Deciduous or semideciduous forest, Ecotone Ombrophilous forest - Deciduous or semi-deciduous forest, Ecotone Savanna (or Brazilian savanna) - Ombrophilous forest, Steppe, Tropical and subtropical semi-deciduous forest, Tropical ombrophilous forest, Subtropical ombrophilous forest, Swamp, Salt meadow, Alpine meadows, Savanna (or Brazilian savanna)

Tropical and subtropical semi-deciduous forest, Subtropical ombrophilous forest, Savanna (or Brazilian savanna)

Tropical and subtropical semi-deciduous forest, Tropical ombrophilous forest

Steppe, Tropical and subtropical semideciduous forest, Tropical ombrophilous forest, Subtropical ombrophilous forest, Swamp, Salt meadow, Alpine meadows, Savanna (or Brazilian savanna)

Ecotone Tropical ombrophilous forest - Subtropical ombrophilous forest, Tropical and subtropical semi-deciduous forest, Subtropical ombrophilous forest

Tropical and subtropical semi-deciduous forest, Savanna (or Brazilian savanna), Steppic-savanna*

Savanna (or Brazilian savanna)

Tropical and subtropical semi-deciduous forest

Tropical and subtropical semi-deciduous forest, Tropical ombrophilous forest, Salt meadow, Savanna (or Brazilian savanna), Steppic-savanna*

Ecotone Savanna (or Brazilian savanna) - Deciduous or semi-deciduous forest, Tropical and subtropical semi-deciduous forest, Steppic-savanna*
Cerrado, Araucaria moist forests

Cerrado, Alto Paraná Atlantic forests, Araucaria moist forests

Araucaria moist forests

Chiquitano dry forests, Araucaria moist forests, Tocantins/Pindare moist forests, Cerrado, Serra do Mar coastal forests

Uruguayan savanna, Alto Paraná Atlantic forests, Cerrado, Araucaria moist forests, Serra do Mar coastal forests, Bahia coastal forests, Atlantic Coast restingas

Alto Paraná Atlantic forests, Cerrado, Araucaria moist forests

Cerrado, Araucaria moist forests

Cerrado, Araucaria moist forests, Alto Paraná Atlantic forests, Uruguayan savanna, Serra do Mar coastal forests, Atlantic Coast restingas

Araucaria moist forests, Uruguayan savanna

Bahia coastal forests, Caatinga

Cerrado

Alto Paraná Atlantic forests

Southern Atlantic mangroves, Pernambuco coastal forests, Bahia coastal forests, Serra do Mar coastal forests, Araucaria moist forests, Caatinga

Caatinga, Pernambuco interior forests, Pernambuco coastal forests, Southern Atlantic mangroves, Maranhão Babaçu forests 


\begin{tabular}{ll}
\hline Family/Species & Physiognomic-ecological class \\
\hline Cortaderia selloana (Schult. \& & Tropical ombrophilous forest, Subtropical \\
Schult. f.) Asch. \& Graebn. & ombrophilous forest \\
Cynodon dactylon (L.) Pers. & Tropical ombrophilous forest, Steppic- \\
& savanna* \\
Digitaria decumbens Stent & Tropical and subtropical semi-deciduous \\
& forest, Steppic-savanna* \\
Echinochloa crus-galli (L.) P. & Swamp
\end{tabular}

Beauv.

Eragrostis plana Nees

Hyparrhenia rufa (Nees) Stapf

Melinis minutiflora P. Beauv.

Melinis repens (Willd.) Zizka

Pennisetum clandestinum Hochst. ex Chiov.

Pennisetum purpureum Schumach.

Urochloa brizantha (Hochst. ex A. Rich.) R. D. Webster

Urochloa decumbens (Stapf) R. D. Webster meadow

Tropical and subtropical deciduous forest, Savanna (or Brazilian savanna)

Ecotone Ombrophilous forest-Deciduous or semi-deciduous forest, Ecotone Savanna (or Brazilian savanna) - Deciduous or semi-deciduous forest, Campinarana*, Steppe, Tropical and subtropical deciduous forest, Tropical and subtropical semideciduous forest, Tropical ombrophilous forest, Subtropical ombrophilous forest, Mangrove forest, Salt meadow, Savanna (or Brazilian savanna), Steppic-savanna* Steppe, Tropical and subtropical deciduous forest, Tropical ombrophilous forest, Salt meadow, Savanna (or Brazilian savanna), Steppic-savanna*

Tropical ombrophilous forest, Salt meadow

Steppe, Tropical ombrophilous forest, Subtropical ombrophilous forest, Salt meadow, Savanna (or Brazilian savanna), Steppic-savanna*

Ecotone Steppe - Deciduous or semideciduous forest, Steppe, Tropical and subtropical semi-deciduous forest, Evergreen broad-leaved woodland, Tropical ombrophilous forest, Salt meadow, Savanna (or Brazilian savanna)
Ecoregion

Araucaria moist forests

Bahia coastal forests, Serra do Mar coastal forests, Caatinga

Caatinga, Atlantic Coast restingas

Cerrado, Caatinga

Steppe, Tropical ombrophilous forest, Cerrado, Uruguayan savanna, Alto Paraná Subtropical ombrophilous forest, Salt Atlantic forests, Bahia coastal forests,

Araucaria moist forests, Bahia interior forests

Araucaria moist forests, Alto Paraná Atlantic forests, Serra do Mar coastal forests, Cerrado, Dry Chaco

Bahia coastal forests

Cerrado, Bahia coastal forests, Araucaria moist forests, Serra do Mar coastal forests, Pantanal, Caatinga

Alto Paraná Atlantic forests, Cerrado, Uruguayan savanna, Pernambuco coastal forests, Pernambuco interior forests, Madeira-Tapajós moist forests, JapuráSolimoes-Negro moist forests, Maranhão Babaçu forests, Tocantins/Pindare moist forests, Atlantic Coast restingas, Caatinga, Purus-Madeira moist forests, Guianan savanna, Atlantic dry forests

Cerrado, Bahia interior forests, MadeiraTapajós moist forests, Southwest Amazon moist forests, Purus-Madeira moist forests, Japurá-Solimoes-Negro moist forests, Bahia coastal forests, Araucaria moist forests, Caatinga, Pernambuco coastal forests, Pernambuco interior forests, Southern Atlantic mangroves, Mato Grosso seasonal forests, Alto Paraná Atlantic forests, Pantanal

continue 
continuation

\begin{tabular}{ll}
\hline Family/Species & Physiognomic-ecological class \\
\hline Urochloa humidicola (Rendle) & Campinarana*, Steppe, Tropical and \\
Morrone \& Zuloaga & subtropical semi-deciduous forest, \\
& Evergreen broad-leaved woodland, \\
& Tropical ombrophilous forest, Salt \\
& meadow, Savanna (or Brazilian savanna), \\
& Steppic-savanna*
\end{tabular}

Ecoregion

Pantanal, Cerrado, Pernambuco coastal forests, Pernambuco interior forests, Purus-Madeira moist forests, Chiquitano dry forests, Madeira-Tapajós moist forests, Southwest Amazon moist forests, JapuráSolimoes-Negro moist forests, Mato Grosso seasonal forests, Uatuma-Trombetas moist forests, Amazon-Orinoco-Southern Caribbean mangroves, Guianan savanna, Maranhão Babaçu forests, Tocantins/ Pindare moist forests, Araucaria moist forests, Bahia coastal forests, Caatinga, Southern Atlantic mangroves, Atlantic dry forests, Atlantic Coast restingas

Urochloa maxima (Jacq.) R. D. Steppe, Tropicaland subtropicaldeciduous Webster forest, Tropical and subtropical semideciduous forest, Tropical ombrophilous forest, Subtropical ombrophilous forest, Salt meadow, Savanna (or Brazilian savanna), Steppic-savanna* Cerrado, Caatinga, Dry Chaco, Bahia interior forests, Bahia coastal forests, Serra do Mar coastal forests, Araucaria moist forests, Tocantins/Pindare moist forests, Chiquitano dry forests, Alto Paraná Atlantic forests, Pantanal

Urochloa mutica (Forssk.) T. Q. Nguyen

Tropical ombrophilous forest, Mangrove forest, Salt meadow

Urochloa plantaginea (Link) R. D. Salt meadow

Araucaria moist forests

Bahia coastal forests

Webster

Urochloa ruziziensis (R. Germ. \&

C. M. Evrard) Crins

Urochloa stolonifera (Gooss.)

Chippindale

Urochloa subquadripara (Trin.) R.

D. Webster

Tropical ombrophilous forest, Swamp

Steppic-savanna*

Campinarana*, Tropical and subtropical semi-deciduous forest, Tropical ombrophilous forest, Swamp, Mangrove forest, Salt meadow, Savanna (or Brazilian savanna)

\section{PROTEACEAE}

Grevillea banksii R. Br.

Grevillea robusta A. Cunn. ex R. Br.

PTERIDACEAE

Pteris vittata $\mathrm{L}$.

RHAMNACEAE

Hovenia dulcis Thunb.
Salt meadow

Steppe, Tropical ombrophilous forest, Swamp, Salt meadow

Steppe

Ecotone Steppe - Deciduous or semideciduous forest, Ecotone Ombrophilous forest - Deciduous or semi-deciduous forest, Ecotone Tropical ombrophilous forest - Subtropical ombrophilous forest, Steppe, Tropicaland subtropicaldeciduous forest, Tropical and subtropical semideciduous forest, Tropical ombrophilous forest, Subtropical ombrophilous forest
Araucaria moist forests

Caatinga

Pantanal, Bahia coastal forests, Cerrado, Araucaria moist forests, Guianan moist forests, Uruguayan savanna, Serra do Mar coastal forests, Atlantic Coast restingas, Alto Paraná Atlantic forests

Bahia coastal forests

Cerrado, Bahia coastal forests, Alto Paraná Atlantic forests

Cerrado

Uruguayan savanna, Alto Paraná Atlantic forests, Araucaria moist forests, Cerrado, Bahia interior forests, Serra do Mar coastal forests 


\begin{tabular}{|c|c|}
\hline Family/Species & Physiognomic-ecological class \\
\hline \multicolumn{2}{|l|}{ ROSACEAE } \\
\hline Eriobotrya japonica (Thunb.) Lindl. & $\begin{array}{l}\text { Ecotone Tropical ombrophilous forest - } \\
\text { Subtropical ombrophilous forest, Steppe, } \\
\text { Tropical and subtropical deciduous forest, } \\
\text { Tropical and subtropical semi-deciduous } \\
\text { forest, Tropical ombrophilous forest, } \\
\text { Subtropical ombrophilous forest }\end{array}$ \\
\hline
\end{tabular}

\section{RUBIACEAE}

Coffea arabica $\mathrm{L}$.

\section{RUTACEAE \\ Citrus aurantium L.}

Citrus limon (L.) Osbeck

\section{THELYPTERIDACEAE \\ Macrothelypteris torresiana \\ (Gaudich.) Ching \\ Thelypteris dentata (Forssk.) E. P. \\ St. John \\ WOODSIACEAE \\ Deparia petersenii (Kunze) M. Kato \\ ZINGIBERACEAE \\ Hedychium coccineum Buch.-Ham. ex Sm.}

Hedychium coronarium J. König
Hedychium gardnerianum Sheppard ex Ker Gawl.

Tropical and subtropical semi-deciduous forest, Tropical ombrophilous forest, Steppic-savanna*

Steppe, Tropical and subtropical semideciduous forest, Tropical ombrophilous forest

Ecotone Ombrophilous forest - Deciduous orsemi-deciduousforest,Steppe,Tropicaland subtropical semi-deciduous forest, Tropical ombrophilous forest, Salt meadow

\section{Steppe Cerrado \\ Steppe Cerrado \\ Steppe Cerrado}

Ecotone Ombrophilous forest Deciduous or semi-deciduous forest, Tropical and subtropical semi-deciduous forest, Tropical ombrophilous forest Ecotone Ombrophilous forest-Deciduous orsemi-deciduous forest, Ecotone Tropical ombrophilous forest - Subtropical ombrophilous forest, Ecotone Savanna (or Brazilian savanna) - Ombrophilous forest, Steppe, Tropical and subtropical deciduous forest, Tropical and subtropical semi-deciduous forest, Evergreen broadleaved woodland, Tropical ombrophilous forest, Subtropical ombrophilous forest, Swamp, Salt meadow, Alpine meadows Subtropical ombrophilous forest
Ecoregion

Araucaria moist forests, Cerrado, Alto Paraná Atlantic forests, Bahia coastal forests, Bahia interior forests, Serra do Mar coastal forests

Alto Paraná Atlantic forests, Araucaria moist forests, Caatinga

Araucaria moist forests, Alto Paraná Atlantic forests, Bahia coastal forests

Cerrado, Alto Paraná Atlantic forests, Araucaria moist forests, Bahia coastal forests, Caatinga, Uruguayan savanna

Araucaria moist forests, Bahia interior forests, Alto Paraná Atlantic forests

Cerrado, Serra do Mar coastal forests, Araucaria moist forests, Bahia interior forests, Caatinga, Alto Paraná Atlantic forests, Bahia coastal forests

Araucaria moist forests

\footnotetext{
${ }^{1}$ Registered in the database as Syzygium malaccense (L.) Merr. \& L.M. Perry
}

stans (239 records), Urochloa humidicola (225 records), Azadirachta indica (220 records), Prosopis juliflora (212 records), Cenchrus ciliaris (194 records), Pinus elliottii (165 records), Leucaena leucocephala (155 records), Pinus taeda (128 records), and Hovenia dulcis (126 records).
However, the invasive plant species covering the largest number of phytophysiognomies are Ricinus communis, Hedychium coronarium, Melinis minutiflora (all present in 12 phytophysiognomies), Pinus elliottii (present in 11), Acacia mearnsii (present in 10), Tecoma 


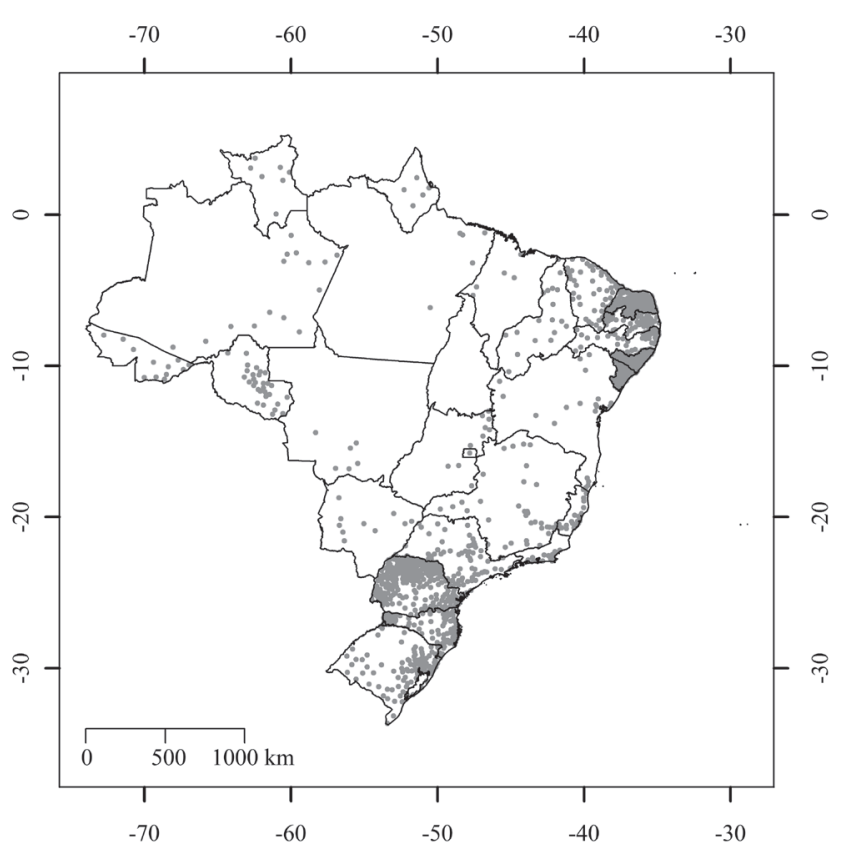

Figure 1. Localities with at least one occurrence record for invasive plants (gray dots) registered in the I3N-Brazil database as of December 2010. Most localities contain only one record for a single species, but some have many. There is likely a strong sampling bias towards the southern and northeastern regions, while a clear lack of information for central and northern regions. Axes are latitude (vertical) and longitude (horizontal).

stans, and Azadirachta indica (both present in 9). Species also worth mentioning due to their relevance as invasives, but present in fewer phytophysiognomies are Artocarpus heterophyllus in tropical ombrophilous forest, Hovenia dulcis in subtropical ombrophilous forest, Prosopis juliflora in Steppic-savanna, and Eragrostis plana in Steppe.

Phytophysiognomies with the highest number of invasive alien plants are, in descending order: tropical ombrophilous forests (69 invasive alien species recorded), tropical and subtropical semi-deciduous forests (60), herbaceous and half-woody salt swamps (45), steppes (40), shrublands or savanna (36) and steppic-savanna (34). As would be expected (figure 1), phytophysiognomies in the Amazon region have the lowest numbers of invasive alien plants recorded (13 species) and very few occurrence records (173 occurrences or $\sim 5 \%$ of the total).

Most documented invasive alien plant species in Brazil are related to human activities and to accessibility by roads, which are greatly lacking in the Northern region. Additionally, many species were intentionally cultivated at some stage even if the original introduction was accidental. Examples includes Eragrostis plana, a plant accidentally introduced but then cultivated as a forage grass, and Cyperus rotundus, cultivated as a medicinal plant.

In order to illustrate the status of biological invasions in Brazil, a few case studies are presented below. We think these case studies also represent a large proportion of the published scientific knowledge available on invasive alien plants for the country.

Eragrostis plana was accidentally introduced as a seed contaminant of Chloris gayana Kunth seed shipments in Rio Grande do Sul State (extreme south of Brazil) in the 1950s. After introduction, E. plana outcompeted the native species Aristida sp. in pastures, which was considered a serious agricultural weed and problem for cattle ranching at the time. Eragrostis plana was therefore considered a viable forage alternative (Reis 1993), which led to large promoting and planting of it as a crop species in Southern Brazil. Only later did ranchers notice that the species was too fibrous when mature. Cattle would either not feed on it (Reis 1993) or would not gain weight when feeding on it. The species is currently established on more than two million hectares of mostly degraded or overgrazed steppes in Rio Grande do Sul state, also occurring in smaller extent in Santa Catarina, Paraná, São Paulo, Mato Grosso, Mato Grosso do Sul, Bahia, Tocantins and Pará and in the Distrito Federal (Medeiros \& Focht 2007), as well as in Uruguay. The species has reported allelopathic effects, especially on species with late germination cycles, from decomposition of plant tissues (Ferreira et al. 2008). It also produces higher root biomass than most native grass species and is more efficient in resource acquisition (i.e. water and nutrients) in soil layers between 0 and $10 \mathrm{~cm}$ (Abichequer et al. 2006). The species is neglected by cattle and thus in highly invaded steppes there are severe economic losses due to the low yield in feeding animals with this forage grass (Marcantonio 2002). Seeds remain viable in the soil more than 24 years when located 20 $\mathrm{cm}$ below the soil surface (Medeiros \& Focht 2007), potentially making the eradication of the species in invaded sites highly unlikely.

Melinis minutiflora was first recorded in Brazil in 1824, after spreading into deforested areas following several human induced fires (Lima 2002). Nowadays this grass is abundant in many areas, especially in the Cerrado (Pivello et al. 1999). The spread of $M$. minutiflora is correlated to a decrease in native species richness in the invaded sites (in experimental plots in the Brasilia National Park the number of native species decreased from 54 in 1997 to 20 in 2001). This has been 
attributed to a positive feedback where $M$. minutiflora increases the fuel loads which increase fire temperatures. When seasonal fires reach these higher temperatures they eliminate the seed bank of most species and allow the invasive grass to dominate the burnt areas (Hoffman et al. 2004, Martins et al. 2004). Moreover, the grass reduces the regeneration of native plants, as shown for Cecropia pachystachya Trécul in riparian areas (Morosini \& Klink 1997).

Native to Mexico and Southern United States, Tecoma stans was introduced in Brazil for landscaping around 1871 and now occupies more than 10,000 hectares of degraded Alto Paraná Atlantic Forests (Bredow et al. 2004). It forms dense thickets and resprouts vigorously. The loss of pastures and agricultural areas to invasion induces farmers to abandon areas and generates an annual loss of US\$ 7,500,000 for the cattle industry (Pedrosa-Macedo 2004). It is forbidden to use, transport or commercialize the species since 1997 in Paraná State (Resolução Estadual no 151/1997).

Prosopis juliflora is a legume tree native to Central America (Palacios 2006) and was introduced as a forage alternative in the 1940's in the Caatinga region, Northeastern Brazil (Pegado et al. 2006). The Brazilian Federal government promoted the distribution of seedlings of P. juliflora in the 1980's, which led to populations scattered across the northeastern states and the Caatinga biome. Some reports note that invaded areas have around $87 \%$ cover of $P$. juliflora, drastically changing the diversity and structure of native forests (Pegado et al. 2006). In invaded riparian areas in Bahia the species represented $60.8 \%$ of the forest regeneration $\left(1,255\right.$ seedlings ha $\left.{ }^{-1}\right)$, followed by another invasive alien plant Parkinsonia aculeata $\mathrm{L}$. which represented $4.3 \%$ ( 89 seedlings ha ${ }^{-1}$ ). Seedlings of other 120 adult native plants occurring in the area shared the other $34.9 \%$ of the forest regeneration (P.C.F. Lima \& L.H.P. Kiill, unpublished data).

Artocarpus heterophyllus is native to high elevation locations (above $1,110 \mathrm{~m}$ ) on the Ghats Mountains in India and the Malay Peninsula (Sahni 2000). It was one of the first alien trees introduced in the Atlantic forest region (Dean 1996). The presence of the species is usually associated with human occupation and it benefits from cultivation as a fruit tree and as an ornamental. Areas invaded by $A$. heterophyllus are found to have higher densities of native rats in comparison to natural forests without the alien plant (Bergallo et al. 2009). Also, the diversity of seed species dispersed by rats also decreases with the increase of $A$. heterophyllus densities (Bergallo et al. 2009). This pattern could lead to a decrease of native plant abundance and to the increase of $A$. heterophyllus dominance over the long term.

\section{Discussion}

Limitations - We found that there is some ability to quantify the species and habitats invaded by reviewing plant occurrence records within the I3N Brazil database. Yet, this database has some limitations in discriminating major and minor invasive plant species within each ecoregion and habitat and across Brazil. Records of occurrences are basically presence records and contain no data on the size and density of occupied areas, or on the dynamics of invasive species at different locations. Therefore, it is not possible to assume that a larger number of records represent a larger area of occurrence. Additionally, as in many records of occurrence in plant databases, it is not possible to determine absences as they are potentially related to the lack of information available or to the presence of the species in a status different than established or invasive (i.e. contained, naturalized, present only in urban areas or unknown).

"Established" refers to species which are reproducing locally, and "invasive" refers to species that are reproducing and spreading beyond their points of introduction. Although this definition may be clear, it is difficult for observers to be sure about the status of invasion in the field, especially as these are often side observations done while performing other work in restoration, animal or plant surveys, or just field expeditions. The lack of expertise on biological invasions in Brazil may also lead to confusion between invasive, established or present species in given habitats. For example, it is crucial to differentiate invasions in well preserved natural habitats from invasions on degraded sites, such as road sides or pastures.

The knowledge available on invasive alien floras and plant species is highly heterogeneous in the country (figure 1), thus comparing species presence and/or invasion status across regions or any other spatial scale is not encouraged. However, historical differences among regions and differences in land use might explain some of the differences in numbers of invasive species. While some regions are more studied than others, with the south and southeast having received more attention than the north and central-west, there is also much variation in the level of ecosystem intactness across the landscape. Also, the number, diversity and intensity of pathways and vectors transporting invasive alien species varies tremendously across regions. 
It is clear that a country as large as Brazil has an enormous challenge assessing its biota due to its size and diversity of ecosystems and landscapes. This overview assessment of plant invasions in Brazil is far from complete or fully accurate. However, it is an initial step towards a better understanding of biological invasions and provides much-needed information for managers and scientists working on invasion issues in Brazil.

Implications for management - We show that, based on a cursory list of invasive species occurring in Brazil, there are many species that have received little to no attention from biologists. Yet, it is clear that invasive alien plants are important components of environmental change in Brazilian ecosystems based on the trend of decreased biodiversity in invaded areas and more research and action is urgently needed to address drivers and consequences of biological invasions. This research will support the implementation of more accurate management and control strategies as well as contribute towards a better theoretical understanding of biological invasions.

Conflicts of interest between those species deemed economically important and those that may prevent the achievement of environmental conservation goals need to be addressed urgently. Both private companies and governmental agencies promote the spread of invasive species (i.e. E. plana for pastures, P. juliflora for cattle and human feeding, and Elaeis guineensis for biofuels) and currently no regulations exist to contain, prevent, or control the spread of species. It is highly desirable that such policies are accompanied by criteria to establish production areas, and that, at the example of certification in Forestry, invasive species that yield economic benefits include prevention and routine control in their management. Some legal limitations are beginning to exist thanks to official invasive species lists issued by the states of Paraná and Santa Catarina, in the south of Brazil, restricting public nurseries to produce seedlings and the use of listed species in restoration and road stabilization efforts.

The country has a fragmented legal framework for dealing with invasive alien species; there are many areas of conflict and many gaps. Existing policies are ineffective in regulating economic activities that are driving the trade of invasive species. Brazilians are beginning to better understand and register the consequences of biological invasions and several promising initiatives are now starting. States are developing strategies for invasive alien species by creating official reference lists of invasive species, controlling and managing invasive alien species in protected areas and restricting the use of these species for certain purposes. The Ministry of Environment published a national strategy for invasive alien species in 2009 with support from a National Committee on Invasive Species involving different Ministries, environmental management and health agencies. This work needs to be supported and continued, aligned with the Convention on Biological Diversity.

It is also important to notice that most of the widely distributed invasive plants in Brazil (such as Pinus spp. and African grasses) are well known for their capacity to thrive under high levels of disturbance. It suggests that several invasions are a consequence of other disturbances rather than agents of disturbance themselves (see Vitousek et al. 1996). Therefore, to maintain the quality of many Brazil's natural ecosystems, it is crucial to prevent and control invasions by alien species.

Implications for Ecology - This study shows that numerous opportunities to study the ecological consequences of invasions in extremely rich, diverse and heterogeneous ecosystems and landscapes exist. These opportunities are currently neglected by biologists and ecologists. Until recently, alien plants were ignored in floristic surveys leading to a lack of historical records and quantitative data on the presence of invasive alien plants in reports and in herbarium and museum collections. Most botanists who contribute material to Herbaria do not bother collecting non-native plants, though these would be precious and useful records for early detection, management and for understanding the spread of invasive species by providing historical records on habitat occupation and spread. For example, many species niche models rely on herbarium records to build species' potential distributions models and to predict suitable habitats for invasive species before they get there (i.e. Zenni et al. 2009).

Many recent ( $<70$ years) plant introductions have led to completely different colonization patterns. While some species became widespread quickly (i.e. Eragrostis plana and Pinus taeda) others are observed as less able to spread (i.e. several Eucalyptus species and Nerium oleander). The causes of these differences are open research questions, as the behavior of the species varies greatly in different climates, ecosystems and parts of the world.

Richardson et al. (2008) recently questioned if pine invasions in South America could become as dramatic as they are in several other Southern Hemisphere countries such as South Africa and New Zealand. We believe the answer to be "yes" from current evidence in a number of countries (Simberloff et al. 2010). However, more 
research on patterns and drivers of pine invasions in Brazil will much contribute to a more precise answer. So far we can only speculate.

There is equal urgency for increasing research, for qualified ecologists and biologists working on the ecology of invasions, for field managers and decisionmakers advancing control work, and for policy-makers supporting legal frameworks in the country and worldwide. Filling the sampling gaps (shown in figure 1) and acquiring knowledge about the dynamics and influences of invasions in different regions and habitats should be invasion biologists' first priority.

Further work - The next steps in invasion efforts should include quantifying invasions of alien plants, evaluating consequences of these invasions on the natural environment, defining priorities and implementing prevention and control strategies when relevant to biodiversity conservation. Thus, it is important that levels of invasion by alien species in different ecosystems are clearly defined as major, minor, incipient, or emerging invaders. Based on these distinctions, priorities should be set according to the potential impacts and the feasibility of eradication or control efforts. The publication of official invasive species lists is an important first step to provide support to managers and institutions to build up prevention and early detection techniques and regional strategies to mitigate the threats posed by biological invasions. Decentralizing these huge task from the federal government to state agencies and to municipalities should greatly help set priorities and understand impacts at the local level, and use these results to multiply the work across the country.

Acknowledgements - We thank everyone who contributed with data and observations during the National Survey on Invasive Alien Species and onward efforts. We thank the Brazilian Ministry of Environment, the Inter-American Biodiversity Information Network (IABIN - I3N), and The Nature Conservancy for funding different parts of this project. We thank Sara Kuebbing for improving the language, Francisco Zenni Guérios for helping with table 1, and two anonymous reviewers for important suggestions.

\section{References}

ABICHEQUER, A.D., MEDEIROS, C.M.O. \& SPANNENBERG, P.R.O. 2006. Crescimento e distribuição de raízes de capim-annoni-2: vantagem competitiva em relação ao campo nativo?. In Anais da 21르 Reunião do Grupo Técnico em Forrageiras do Cone Sul. Embrapa Clima Temperado, Pelotas, CD-ROM.
BERGALLO, H.G., RAÍCES, D.S.L., UZÊDA, M.C., ROCHA, C.F.D., ABREU, R.C. \& FERREIRA, P.M. 2009. Densidade de jaqueiras e seus efeitos na comunidade de pequenos mamíferos, dispersão de sementes e agregados de solo na Ilha Grande, RJ. In Anais do I Congresso Brasileiro sobre Bioinvasão. Universidade Federal do Maranhão, São Luís, CD-ROM.

BREDOW, E.A., PEDROSA-MACEDO, J.H. \& VITORINO, M.D. 2004. Plantas invasoras no Paraná. In Princípios e rudimentos do controle biológico de plantas (J.H. Pedrosa-Macedo \& E.A. Bredow, eds.). Universidade Federal do Paraná, Curitiba, p.51-99.

COOK,B.G.,PENGELLY,B.C.,BROWN, S.D.,DONNELLY, J.L., EAGLES, D.A., FRANCO, M.A., HANSON, J., MULLEN, B.F., PARTRIDGE, I.J., PETERS, M. \& SCHULTZE-KRAFT, R. 2005. Tropical forages: an interactive selection tool. http://www.tropicalforages. info (accessed 2009 Mar 25).

DEAN, W. 1996. A ferro e fogo: a história e a devastação da mata atlântica brasileira. Companhia das Letras, São Paulo.

FERREIRA, N.R., MEDEIROS, R.B. \& SOARES, G.L.G. 2008. Potencial alelopático do capim-annoni-2 (Eragrostis plana Nees) na germinação de sementes de gramíneas perenes estivais. Revista Brasileira de Sementes 30:43-50.

HOFFMANN, W.A., LUCATELLI, V.M.P.C., SILVA, F.J., AZEVEDO, I.N.C., MARINHO, M.S., ALBUQUERQUE, A.M.S., LOPES, A.O. \& MOREIRA, S.P. 2004. Impact of the invasive alien grass Melinis minutiflora at the savanna-forest ecotone in the Brazilian Cerrado. Diversity and Distributions 10:99-103.

I3N-BRASIL. 2010. Base de dados sobre espécies exóticas invasoras. http://www.institutohorus.org.br (accessed 2010 Dez 20).

LIMA, M.E.A.T. 2002. As caminhadas de Auguste de SaintHilaire pelo Brasil e Paraguai. Editora Autêntica, Belo Horizonte.

MACK, R.N., SIMBERLOFF, D., LONSDALE, W.M., EVANS, H., CLOUT, M. \& BAZZAZ, F.A. 2000. Biotic invasions: causes, epidemiology, global consequences, and control. Ecological Applications 10: 689-710.

MARCANTONIO, G. 2002. Manjedoura cincerro. Federacite, Porto Alegre.

MARTINS, C.R., LEITE, L.L. \& HARIDASAN, M. 2004. Capim-gordura (Melinis minutiflora P. Beauv.), uma gramínea exótica que compromete a recuperação de áreas degradadas em unidades de conservação. Revista Árvore 28:739-747.

MEDEIROS, R.B. \& FOCHT, T. 2007. Invasão, prevenção, controle e utilização do capim-annoni-2 (Eragrostis plana Nees) no Rio Grande do Sul, Brasil. Revista Agropecuária Gaúcha 13:1-28. 
MEYERSON, L.A. \& MOONEY, H.A. 2007. Invasive alien species in an era of globalization. Frontiers in Ecology and the Environment 5:199-208.

MOROSINI, I.B.A. \& KLINK, C.A. 1997. Interferência do capim-gordura (Melinis minutiflora Beauv.) no desenvolvimento de plântulas de embaúba (Cecropia pachystachya). In Contribuição ao conhecimento ecológico do cerrado (L.L. Leite \& C.H. Saito, org.). Congresso de Ecologia do Brasil. Universidade Federal de Brasília, Brasília, CD-ROM.

MYERS, N., MITTERMEIER, R.A., MITTERMEIER, C.G., FONSECA, G.A.B. \& KENT, J. 2000. Biodiversity hotspots for conservation priorities. Nature 403:853858.

OLSON, D.M., DINERSTEIN, E., WIKRAMANAYAKE, E.D., BURGESS, N.D., POWELL, G.V.N., UNDERWOOD, E.C., D'AMICO, J.A., ITOUA, I., STRAND, H.E., MORRISON, J.C., LOUCKS, C.J., ALLNUTT, T.F., RICKETTS, T.H., KURA, Y., LAMOREUX, J.F., WETTENGEL, W.W., HEDAO, P. \& KASSEM, K.R. 2001. Terrestrial ecoregions of the world: a new map of life on Earth. Bioscience 51: 933-938.

PALACIOS, R.A. 2006. Los mezquites mexicanos: biodiversidad y distribución geográfica. Boletín de la Sociedad Argentina de Botánica 41:99-121.

PEDROSA-MACEDO, J.H. 2004. Plantas invasoras no Paraná. In Princípios e rudimentos do controle biológico de plantas (J.H. Pedrosa-Macedo \& E.A. Bredow, eds.). Universidade Federal do Paraná, Curitiba, p.115-128.

PEGADO, C.M.A., ANDRADE, L.A., FÉLIX, L.P. \& PEREIRA, I.M. 2006. Efeitos da invasão biológica de algaroba - Prosopis juliflora (Sw.) DC. sobre a composição e a estrutura do estrato arbustivo-arbóreo da caatinga no município de Monteiro, PB, Brasil. Acta Botanica Brasilica 20:887-898.

PETENON, D. \& PIVELLO, V.R. 2008. Plantas invasoras: representatividade da pesquisa dos países tropicais no contexto mundial. Natureza \& Conservação 6:65-77.

PIVELLO, V.R., SHIDA, C.N. \& MEIRELLES, S.T. 1999. Alien grasses in Brazilian savannas: a threat to the biodiversity. Biodiversity and Conservation 8 : 1281-1294.
REIS, J.C.L. 1993. Capim annoni-2: origem, morfologia, características, disseminação. In Reunião regional de avaliação de pesquisa com annoni-2. Embrapa-CPPSUL, Bagé, v.1., p.5-23.

RICHARDSON, D.M., VAN WILGEN, B.W. \& NUÑEZ, M.A. 2008. Alien conifer invasions in South America: short fuse burning? Biological Invasions 10:573-577.

SAHNI, K.C. 2000. The book of Indian trees. Oxford University Press, Delhi.

SIMBERLOFF, D., NUÑEZ, M.A., LEDGARD, N.J., PAUCHARD, A., RICHARDSON, D.M., SARASOLA, M., VAN WILGEN, B.W., ZALBA, S.M., ZENNI, R.D., BUSTAMANTE, R., PEÑA, E. \& ZILLER, S.R. 2010. Spread and impact of introduced conifers in South America: lessons from other southern hemisphere regions. Austral Ecology 35:489-504.

TRAVESET, A. \& RICHARDSON, D.M. 2006. Biological invasions as disruptors of plant reproductive mutualisms. Trends in Ecology and Evolution 21:208-216.

UNESCO. 1973. International Classification and Mapping of Vegetation. Ecology and conservation, v.6. United Nations Educational, Scientific and Cultural Organization, Paris.

VELOSO, H.P., OLIVEIRA FILHO, L.C., VAZ, A.M.S.F., LIMA, M.P.M., MARQUETE, R. \& BRAZÃO, J.E.M. 1992. Manual técnico da vegetação brasileira. Manuais técnicos em geociências, v.1. Fundação Instituto Brasileiro de Geografia e Estatística, Rio de Janeiro.

VITOUSEK, P.M., D'ANTONIO, C.M., LOOPE, L.L. \& WESTBROOKS, R. 1996. Biological invasions as global environmental change. American Scientist 84:468-478.

WILCOVE, D.S., ROTHSTEIN, D., DUBOW, J., PHILLIPS, A. \& LOSOS, E. 1998. Quantifying threats to imperiled species in the United States. Bioscience 48: 607-615.

WITTENBERG, R. \& COCK, M.J.W. 2001. Invasive alien species: a toolkit of best prevention and management practices. CAB International, Wallingford.

ZENNI, R.D., WILSON, J.R.U., LE ROUX, J.J. \& RICHARDSON, D.M. 2009. Evaluating the invasiveness of Acacia paradoxa in South Africa. South African Journal of Botany 75:485-496. 